\title{
POLYNOMIAL APPROXIMATION ON A CURVE OF THE FOURTH DEGREE*
}

\section{BY DUNHAM JACKSON}

1. Introduction. In the study of orthogonal polynomials and polynomial approximation, or of the corresponding theory for trigonometric sums, a powerful auxiliary is Bernstein's theorem on the derivative of a trigonometric sum or of a polynomial. When it is desired to investigate similar problems relating to approximation by means of polynomials on a curve in the plane of two real variables $\dagger x$ and $y$, the question arises whether something in the nature of Bernstein's theorem is available in this case also. For some of the simplest curves, such as a line segment or a circle, the question is merely one of interpretation, the polynomials in $x$ and $y$ reducing at once to polynomials or trigonometric sums in terms of the arc length (or a constant multiple of it) as parameter. For any curve segment of the form $x=\phi(t)$, $y=\psi(t)$, where $\phi(t)$ and $\psi(t)$ are polynomials in $t$, there is an immediate answer as far as differentiation with respect to $t$ is concerned; and if $\left|\phi^{\prime}(t)\right|+\left|\psi^{\prime}(t)\right|$ is everywhere positive (and so has a positive lower bound) on the closed range of values of $t$ considered, a derivative with respect to arc length does not exceed a constant multiple of the derivative with respect to $t$. This paper is concerned with an illustrative case in which the problem appears to be not entirely trivial, and yet susceptible of simple and elementary treatment. An appropriate form of Bernstein's theorem is obtained, and its application to a problem of polynomial approximation is indicated.

A concluding paragraph relates to the convergence in the mean of developments in series of orthogonal polynomials on an arbitrary curve.

2. Bernstein's Theorem. Let $C$ be the curve

$$
x^{4}+y^{4}=1 \text {. }
$$

\footnotetext{
* Presented to the Society, December 31, 1936.

$\dagger$ See D. Jackson, Orthogonal polynomials on a plane curve, presently to appear in the Duke Mathematical Journal.
} 
It can be represented parametrically by the equations

$$
x=|\cos \theta|^{1 / 2} \operatorname{sgn} \cos \theta, \quad y=|\sin \theta|^{1 / 2} \operatorname{sgn} \sin \theta .
$$

Let $s$ be the arc length, measured counter-clockwise from the point $(1,0)$. Let $P(x, y)$ be a polynomial of the $n$th degree in the variables $x$ and $y$ together, and let

$$
|P(x, y)| \leqq L
$$

at all points of the curve. It is to be shown that

$$
\left|\frac{d}{d s} P(x, y)\right| \leqq A n L
$$

everywhere on the curve, $A$ being an absolute constant.

Let

$$
\begin{aligned}
& \alpha_{1}(x, y)=\frac{1}{4}[P(x, y)+P(-x, y)+P(x,-y)+P(-x,-y)], \\
& \beta_{1}(x, y)=\frac{1}{4}[P(x, y)-P(-x, y)+P(x,-y)-P(-x,-y)], \\
& \gamma_{1}(x, y)=\frac{1}{4}[P(x, y)+P(-x, y)-P(x,-y)-P(-x,-y)], \\
& \delta_{1}(x, y)=\frac{1}{4}[P(x, y)-P(-x, y)-P(x,-y)+P(-x,-y)] .
\end{aligned}
$$

Each of these polynomials has $L$ as an upper bound for its absolute value on $C$. They are respectively of the form

$$
\begin{array}{ll}
\alpha_{1}(x, y)=\alpha_{2}\left(x^{2}, y^{2}\right), & \beta_{1}(x, y)=x \beta_{2}\left(x^{2}, y^{2}\right), \\
\gamma_{1}(x, y)=y \gamma_{2}\left(x^{2}, y^{2}\right), & \delta_{1}(x, y)=x y \delta_{2}\left(x^{2}, y^{2}\right),
\end{array}
$$

where $\alpha_{2}, \beta_{2}, \gamma_{2}, \delta_{2}$ are polynomials of degree not exceeding $n / 2$ and so (for $n \geqq 1$ ) not exceeding $n-1$, in the two arguments together.

Since a polynomial in $x^{2}$ and $y^{2}$ is a polynomial in $|\cos \theta|$ and $|\sin \theta|$ and consequently, for $0 \leqq \theta \leqq \pi / 2$, a trigonometric sum in $\theta$, the expressions $\alpha_{1}, \cdots, \delta_{1}$ can be written as

$$
\begin{array}{ll}
\alpha_{1}(x, y)=\alpha(\theta), & \beta_{1}(x, y)=(\cos \theta)^{1 / 2} \beta(\theta), \\
\gamma_{1}(x, y)=(\sin \theta)^{1 / 2} \gamma(\theta), & \delta_{1}(x, y)=(\sin \theta \cos \theta)^{1 / 2} \delta(\theta)
\end{array}
$$


for $0 \leqq \theta \leqq \pi / 2$, where $\alpha(\theta), \cdots, \delta(\theta)$ are trigonometric sums of order not greater (and in general considerably less) than $n-1$. From extensions of Bernstein's theorem established elsewhere* it follows that the absolute value of the derivative of each of the last four right-hand members with respect to $\theta$ has for $0<\theta<\pi / 2$ an upper bound of the form

$$
\frac{A_{1} n L}{(\sin \theta \cos \theta)^{1 / 2}}
$$

where $A_{1}$ is an absolute constant; it is to be noted that as regards the orders of magnitude concerned $(\sin \theta)^{1 / 2},(\cos \theta)^{1 / 2}$ are equivalent to $\theta^{1 / 2},(\pi / 2-\theta)^{1 / 2}$.

On passage to the second, third, or fourth quadrant the trigonometric sums $\alpha, \cdots, \delta$ are modified by substitution of $-\cos \theta$ for $\cos \theta$, or of $-\sin \theta$ for $\sin \theta$, or both, in the polynomial expressions, but the form of the conclusion is unchanged except for the replacement of $\sin \theta$ and $\cos \theta$ by their absolute values. Since $P(x, y)=\alpha_{1}+\beta_{1}+\gamma_{1}+\delta_{1}$, there is an absolute constant $A_{0}$ such that

$$
\left|\frac{d}{d \theta} P(x, y)\right| \leqq \frac{A_{0} n L}{|\sin \theta \cos \theta|^{1 / 2}}
$$

in all four quadrants.

For $0<\theta \leqq \pi / 4$,

$$
\frac{d s}{d \theta} \geqq \frac{d y}{d \theta}=\frac{\cos \theta}{2(\sin \theta)^{1 / 2}} \geqq \frac{2^{-3 / 2}}{(\sin \theta)^{1 / 2}} \geqq \frac{2^{-7 / 4}}{(\sin \theta \cos \theta)^{1 / 2}},
$$

and the relation $d s / d \theta \geqq|d x / d \theta|$ leads to the same result for $\pi / 4 \leqq \theta<\pi / 2$. So

$$
\frac{d \theta}{d s} \leqq 4(\sin \theta \cos \theta)^{1 / 2}
$$

throughout the interval $(0, \pi / 2)$. The same conclusion, with $|\sin \theta \cos \theta|^{1 / 2}$ in the right-hand member, holds in the other quadrants also. It follows that

* See D. Jackson, Bernstein's theorem and trigonometric approximation, Transactions of this Society, vol. 40 (1936), pp. 225-251; Theorems 1, 3, 4. 


$$
\left|\frac{d}{d s} P(x, y)\right| \leqq 4 A_{0} n L
$$

everywhere on the curve, in agreement with the assertion that was to be proved.*

3. Convergence. This proposition can be used in connection with the theory of closest polynomial approximation on the curve $C$ just as the standard form of Bernstein's theorem is used in the theory of approximation by trigonometric sums. $\dagger$

For detailed interpretation of the results information is needed as to the possibility of representing a given function of $s$ on $C$ by means of polynomials in $x$ and $y$ with a specified degree of accuracy. The discussion here will be restricted to a simple case illustrating the accessibility of the question to treatment.

Let $s$ be measured counter-clockwise from the point $(1,0)$, as already suggested, let $\sigma$ be the total length of the curve, and let $f(s)$ be a continuous function of period $\sigma$ satisfying a Lipschitz condition with respect to $s$. Let a function $F(x, y)$ be defined throughout the $(x, y)$-plane by the specifications that it shall vanish at the origin, shall be linear on each ray issuing from the origin, and on each of these rays shall coincide in value with $f(s)$ at the point where the ray meets the curve. Then $F(x, y)$ satisfies a Lipschitz condition as a function of the two variables.

(This observation, evident by geometric intuition applied to the surface $z=F(x, y)$, may be analyzed as follows. If $O$ is the origin, and if $P$ and $Q$ are two points lying on a curve $x^{4}+y^{4}$ $=$ constant, subject to the restriction that the magnitude of the angle $P O Q$ does not exceed $\pi / 2$, say, the ratio of the arc $P Q$ to the chord $P Q$ does not exceed a constant. Also, the angle $P Q O$ has a positive lower bound and an upper bound less than $\pi$. Let $P$ and $R$ be any two points of the plane for which the magnitude of the angle $P O R$ is not greater than $\pi / 2$. Let $Q$ be the point in which the ray $O R$ meets that curve $x^{4}+y^{4}=$ constant which

* Although the proof does not apply in the first instance at the quadrantal points where $\sin \theta \cos \theta=0$, the validity of the conclusion at these points follows by continuity.

† See, for example, D. Jackson, Certain problems of closest approximation, this Bulletin, vol. 39 (1933), pp. 889-906; Lemmas 1 and 5, Theorems 1, 2, 9, and 10. 
passes through $P$. In the (rectilinear) triangle $P Q R$, we have $P Q: P R=\sin R: \sin Q, Q R: P R=\sin P: \sin Q ;$ as $\sin Q=\sin P Q O$ has a positive lower bound, each of the distances $P Q, Q R$ is less than a constant multiple of $P R$. The ratio of $|F(P)-F(Q)|$ to the length of the arc $P Q$ is the same as for the corresponding points on the given curve $x^{4}+y^{4}=1$, and so does not exceed the constant of the Lipschitz condition of the hypothesis. Hence $|F(P)-F(Q)|$ is not greater than a constant multiple of the chord $P Q$, and not greater than a constant multiple of $P R$. On the ray $O R$, where $F$ is a linear function of distance with coefficient less than or equal to the corresponding value of $|f(s)|,|F(Q)-F(R)|$ does not exceed a constant multiple of $Q R$, because of the boundedness of $f(s)$, and so is not greater than a constant multiple of $P R$. Therefore $|F(P)-F(R)|$ does not exceed a constant multiple of $P R$. If the angle $P O R$ is greater than $\pi / 2,|F(P)-F(O)|$ does not exceed a constant multiple of $O P,|F(O)-F(R)|$ does not exceed a constant multiple of $O R$, each of the distances $O P, O R$ is less than $P R$, and $|F(P)-F(R)|$ again does not exceed a constant multiple of the distance $P R$.)

Consequently* there exist polynomials $P_{n}(x, y)$, of the $n$th degree in $x$ and $y$, approximating $F(x, y)$ throughout the square $-1 \leqq x \leqq 1,-1 \leqq y \leqq 1$, say, with maximum error not exceeding a constant multiple of $1 / n$. In particular $f(s)$, to which $F(x, y)$ reduces on $C$, is represented by polynomials of the $n$th degree in $x$ and $y$ with the same upper bound for the magnitude of the error.

Taken in conjunction with the theorem of the first part of the paper, by the use of a type of argument referred to above as employed elsewhere in the theory of approximation by trigonometric sums, this means that under the hypothesis that has been imposed on $f(s)$ if polynomials $P_{n}(x, y)$ of degree $n$ are determined for successive values of $n$ so as to minimize the integral

$$
\int_{C}\left|f(s)-P_{n}(x, y)\right|^{m} d s,
$$

* See, for example, D. Jackson, Über die Genauigkeit der Annäherung stetiger Funktionen... , Dissertation, Gottingen, 1911, pp. 16-17, 88; E. L. Mickelson, On the approximate representation of a function of two variables, Transactions of this Society, vol. 33 (1931), pp. 759-781, §5. 
with $m>1$, the polynomials $P_{n}(x, y)$ will converge uniformly on $C$ toward $f(s)$. In particular, for $m=2$, the expansion of $f(s)$ in series of polynomials orthogonal on $C$, with parameter $s$ and weight function 1 , will converge uniformly toward $f(s)$. The statements can be generalized immediately by introduction of a suitably restricted weight function in the integral to be minimized.

4. Convergence in the Mean. As regards the convergence of developments in series of orthogonal polynomials, the proof just presented suggests an approach to the problem of convergence in the mean, not merely for the special curve which has been under consideration, but for an arbitrary curve, with an arbitrary parametric representation. Let a curve $C$ (algebraic or non-algebraic) be given by a pair of equations $x=\phi(t), y=\psi(t)$, where $\phi$ and $\psi$ are continuous for $0 \leqq t \leqq a$ (or continuous everywhere and of period $a$ ), and no two distinct values of $t$ (or no two distinct values in a period) correspond to a single point $(x, y)$, and let a corresponding system of orthogonal polynomials be constructed as described in the paper to which reference is made in the footnote to the opening paragraph above, the weight function, for simplicity of statement, being taken as 1 . If $f(t)$ is any function continuous over the range of $t$, a function $F(x, y)$ can be formed* which is continuous in the two variables throughout a rectangle containing $C$, and reduces on $C$ to $f(t)$. This $F(x, y)$ can be uniformly approximated throughout the rectangle by polynomials in $x$ and $y$ with any degree of accuracy, and on the curve $C$ in particular $f(t)$ is uniformly approximated, and so approximated in the mean, by the same polynomials. It follows that any function $g(t)$ which is of class $L^{2}$ for $0 \leqq t \leqq a$ can be approximated in the mean with index 2 by polynomials in $x$ and $y$, and hence by the least-square property that the development of $g(t)$ in series of orthogonal polynomials converges in the mean with this index.

The University of Minnesota

* See Hassler Whitney, Analytic extensions of differentiable functions defined in closed sets, Transactions of this Society, vol. 36 (1934), pp. 63-89, p. 63; also, for example, D. Jackson, An elementary boundary value problem, this Bulletin, vol. 22 (1915-16), pp. 393-397. 